\title{
Human factors identification and classification related to accidents' causality on hand injuries in the manufacturing industry
}

\author{
Rosa María Reyes-Martínez ${ }^{1}$, Aide Maldonado-Macías ${ }^{\mathrm{a}, \mathrm{b}}$ and Lilia Roselia Prado-León ${ }^{\mathrm{c}}$ \\ ${ }^{a}$ Undergraduate Division Studies, Juarez Institute of Technology, 1340 Tecnológico Avenue, Cd. Juárez, \\ Chihuahua, México, \\ ${ }^{\mathrm{b}}$ Industrial Engineering Department, Autonomous University of Juárez City, 430 Del Charro Avenue North, Cd. \\ Juárez, Chihuahua, México. \\ ${ }^{c}$ University Center of Health Sciences, University of Guadalajara City, 950 Sierra Mojada Avenue, Guadalajara \\ Jalisco, México.
}

\begin{abstract}
The causes of occupational accidents from the perspective of human factors have been a subject which has received little attention into the field of scientific research. The aim of this research was to identify and classify the human factors that influence human errors and failures that cause accidents and injuries specifically on hands. Available studies related to the topic have been developed mainly for aerospace applications and are found insufficient to explain accidents causalities in the manufacturing industry. This research was developed in the assembly industry of automotive harnesses and was conducted following a mixed Cognitive Anthropological approach. This study was developed in two phases. During the first qualitative phase, participants freely listed their knowledge to identify elements of the cultural domain, then and in the second phase they performed the successive pile sort technique for the collection data to classify elements in the cultural domain. Statistical models like Cluster Analysis and Multidimensional Scaling were applied for results' validation purposes. As results, 70 different human factors were identified and in the second phase they were classified into 4 main categories which were: human error, unsafe conditions, individual factors, and organizational factors. Statistical methods validated these results.
\end{abstract}

Keywords: Keyword one, keyword two, keyword three, keyword four, keyword five

\section{Introduction}

The Health and Safety Executive (HSE) describes human factors such as the scientific discipline that concerns the study of human cognitive and physical needs on their interaction with the work environment, their influence on equipment and system design and on human performance [15]. The identification and study of human factors is of particular interest for the field of safety as these factors are often involved in human errors. Their field of knowledge is vast since it "studies the interaction between people, technology and work, with the major aim to find areas where design and working conditions produce human error" [13].

Human error analyses and their role in accidents causalities is an important part of developing syste- matic methodologies for reliability in the industry and for risk prediction. In order to obtain data for predictive analysis, is necessary to analyze accidents and incidents to identify its causes in terms of component of failures and human errors [17].Therefore, a proper understanding of human factors in the workplace is an important aspect in the prevention of accidents [2], and human factors should be considered in any program to prevent those that are caused by human error.

The first step in any program of accidents prevention is error identification and classification, which may allow appropriate prevention and mitigation strategies to be developed for this goal .Taxonomies are used for human error identification and classification [35].

\footnotetext{
${ }^{1}$ Corresponding autor. E-mail:rosyreyes2001@yahoo.com
} 
The dominant theoretical approach for studying the root causes of accidents is known as the "Swiss Cheese" model [19], it proposes that the fundamental components of an organization must work harmoniously for a safe and efficient production system. Although the model provides a unifying framework for studying root causes, researchers and safety practioners have questioned the effectiveness of applying this model to the study of accidents. Wiegmann and Shappell noted that Reason's model "fails to identify the exact nature of the 'holes' in the cheese" [25]. The model tends to be theoretical and less analytical, making it difficult to apply.

In response to this challenge, Shappell and Wiegmann developed the Human Factors Classification System (HFACS) that utilizes a systemic approach. The HFACS was originally developed to be used for the United States Navy and Marine Corps [11]. This framework defines the holes in the cheese, drawing upon concepts of latent and active failures; it describes four levels of failure that correspond to Reason's model framework: (a) unsafe acts of operators, (b) preconditions for unsafe acts, (c) unsafe supervision, and (d) organizational influences [19]. In other words, the HFACS goes beyond the simple identification of what an operator did wrong, and provide a clear understanding of the reasons of error occurrence in the first place.

HFACS has been utilized intensively in investigating accidents in high risk systems such as aviation $[11,32]$, in emergency medical transport accidents [1], railway accident transportation [33, 35], healthcare practice [14] and surgery operations [8]. For the maritime industry, the scope of the existing HFACS has been modified and extended to identify the influence of system hardware on human errors in shipping accidents. Recently, HFACS was also proposed as a means to reduce occupational accidents in Turkish shipyards [24]. However, HFACS has been found insufficient to explain accidents causalities in the manufacturing industry, specifically in the assembly industry of automotive harnesses.

This research was developed in the assembly industry of automotive harnesses and was conducted following a combined Cognitive Anthropological approach. The main objective of this research was to identify and classify those human factors that influence human errors and failures and are cause of accidents and injuries specifically in the hands. The main theoretical framework of this work is presented below.

\section{Cognitive Anthropology}

The cognitive anthropology provides a helpful theoretical orientation in the study of culture. It suggests that culture is composed of an interconnected framework of schematized, shared knowledge that constructs meaning, represents social reality, directs behavior, and facilitates the interpretation of behavior $[27,28]$. Cognitive anthropological researchers have developed methods to minimize the etic (researcher-determined) and maximize the emic (participant-determined) aspects of the research and thus mix qualitative and quantitative data collection techniques in that goal [7].In this case this field of knowledge provided the mixed methods for the study of human factors identification and their association to accidents causalities on hand injuries.

\subsection{Cultural Consensus Theory}

The cognitive anthropologists Romney, Weller and Batchelder developed the Cultural Consensus Theory (CCT) in 1986 [6].CCT is a collection of analytical techniques and models that can be used to estimate cultural beliefs and the degree by which individuals know or report those beliefs. CCT estimates the culturally correct answers for a series of questions (group of beliefs) and simultaneously estimates each respondent's knowledge or degree of correspondence among the answers [34].

The cultural consensus model was created to offer field researchers with objective approximation of the distribution of shared knowledge among informants in relation to a specified cultural domain. $[4,5,6]$ The theory employs the pattern of agreement or consensus among informants to supply an estimate of the cultural competence or knowledge of each informant and an estimate of the culturally correct answer to each question asked of the informants the consensual answer key.

A domain may be defined as an organized set of words, concepts, phrases and sentences, all on the same level of contrast, that jointly refer to a single conceptual sphere [31].

For C.Webster et al. [10] this theory has been applied to a diverse of domains as: disease classification [6], causes of fatalities, [4], occupational prestige [5], semiotic models of alphabetic systems [21, 22], illness beliefs of deaf senior citizens [9], knowledge of high blood pressure [23], and classification of animals and student behaviors relating to good grades [12]. In this case a novel application is made 
for human factors classification for accidents' causalities on hand injuries in the manufacturing industry.

\section{Methods}

The scientific methodology used in this study corresponds to a Cognitive Anthropology and mixed methods approach, with the application of an informal version Cultural Consensus Theory. The data collection techniques such as free listing and pile sort (card sorting) were used with cultural domain methods analysis described by Weller and Romney [34] and Ross [3]. These techniques were applied sequentially, that is, each subsequent data collection step was based on the findings from the preceding step.

The study was completed via two stages. The first one identifies cultural domain elements of human factors from a cultural domain analysis that begins with open-ended interviews. These consisted of structured interviews, and somewhat more specialized interviews such as the free list. The aim of each analysis is to generate the terms that individuals use to talk about a particular cultural domain, which in this case are human factors related to accidents. The purpose in second phase was sorting human factors elements with mixed methods. In the unconstrained pile sort, the participant simply indicates how terms are grouped together on the basis of similarity in meaning and separated from other terms in other groups on the basis of differences in meaning. These methods are described in the following sections.

\subsection{Identification of human factors in accidents}

Human factors related to accidents 'causality on hand injuries were identified by cultural domain items, a structured interview known as free listing was used to collect data. Free listing is a common elicitation technique and it is one of the best ways to ensure that the concepts and the domain are culturally relevant. It provides a strong source of cognitive data in terms of frequencies [34].

The free listing was structured with five questions, the generating terms used were: human error, unsafe conditions, personal factors, organizational failures and management failures. When free listing are used, it is common practice to write words, but due of the nature and complexity of accident causation presen- ted; participants were asked to freely list phrases and sentences related to accident causality terms in each question.

\subsubsection{Characteristics of the sample}

The inclusion criteria considered for participants was: having seniority greater than 10 years, be a member of the industrial safety group, having knowledge about accidents' occurrence in the company and the willingness to participate in this stage of the study.

The non probabilistic sampling strategy of snowball was used for selecting participants; key informants were production superintendent, human resources manager and safety coordinator who provided a list of 30 persons belonging to both work shifts (morning and evening), all of them satisfied inclusion criteria. They all were invited to participate, but only 21 of them completed the free listing.

According to Weller and Romney a sample of 20 to 30 informants can be adequate. A larger or smaller sample would be necessary depending on the degree of agreement of responses. Even a sample size of 20 is assumed to be adequate $[31,34]$. The sample was non-probabilistic, flexible and representative of the safety group, its size was of 21 and it was integrated by supervisors of: manufacturing (six), maintenance (three), human resources (one), quality reliability (one) and managers of the following areas: training (one), manufacturing ( (two), engineering (one), occupational health (one), methods engineer (one) and quality (one); technical level was represented by one employee of health and safety and another to environment, also the manufacturing superintendent was part of the sample.

\subsubsection{Determination of Cultural Domain}

The cultural domain was obtained through the free listing technique. In order to avoid data's bias, free listing responses were tabulated verbatim. The decision about how many items can be included in the domain is determined following several considerations: the purpose of the study, the number and frequency of the listed. These items were tabulated in a frequency table in a descendent order. Then, to ensure that the items included in the previous list were known among informants and avoid unwanted biases, a second free listing with false and true questions was applied. In this, all items whose frequen- 
cies were less than $50 \%$ were included. From a total of 21 informants who applied to the first free listing, only 17 of them were located in the workplace and interviewed by the researcher to complete the second free listing.

\subsection{Classification of human factors in accidents}

The cultural domain items obtained through free listing were selected in order to classify those human factors related to accidents. A successive pile sort technique was used for data collection. Informants classified seventy cards designed for this purpose; each card presented one phrase or sentence which describes a particular human factor.

In the application of the successive pile sort technique a top-down approach was selected. The seventy cards were classified in piles according to similarity of the criterion established by the informants' agreement. Participants agreed on the similarity criterion to classify human factors that influence accidents in two ways: the first related to personal factors and the other with organizational factors.

The sample size in pile sort technique usually can be between 20-30 informants, although the number of participants depends on the amount of variability of responses [30,31,34]. In this case, only 12 informants were included, this can be adequate due to variability in responses was very small.

Data analysis was performed using multivariate techniques such as: Cluster Analysis, Multidimensional Scaling (MDS) and factor analysis (Cultural Consensus Analysis). In order to select the best model that described the human factors influence accidents with hands injury, the above techniques were applied to cases with 2, 4 and 8 piles.

The results of the pile sort task were analyzed using hierarchical cluster analysis with a non metric version. For each informant, a $70 \times 70$ matrix was created based on their card-sorting data. These data were transformed to distance scores and then aggregated across informants to create a composite distance matrix that was used in the cluster analysis.

The average linkage inter-groups algorithm was selected for conducting the cluster analysis because it is often used for clustering cards to develop categories. This method is attractive because it associates a factor with a cluster if it is similar on average to the other factors in the cluster [16 ]. The Chisquare frequency value was used as a measure of similarity using the Statistical Package for Social
Sciences (SPSS) version 15 for Windows XP for data processing.

The most common technique for visualizing perceived similarities or dissimilarities is MDS. It creates a graphical representation of a square itemby-item proximity matrix. In cultural domain analysis, the input matrix is an aggregate similarity matrix, which represents the proportion of times that a given pair of items was seen as similar by respondents in elicitation tasks such as pile sorts [26].

The non metric MDS was applied in this study; the Visual Anthropac statistical package version 2.1.3.5 developed by Stephen Borgatti [30] was used in data processing.

The purpose of cultural consensus analysis was used to verify the existence of a shared safety culture among members of the security group. This was performed according to the informal cultural consensus model, using a factorial analysis of the participants (principal component analysis). The statistical package version 1.2.35 Visual Anthropac was used in information processing.

In addition, the cultural consensus analysis was used to demonstrate the existence of a coherent cultural domain among participants, the level of agreement among members of the security group and the cultural competence coefficients .The culturally correct knowledge of each informant related to human factors that influence human error in accidents, which an adverse result is hands injury were estimated by cultural competence coefficients.

\section{Results}

\subsection{Participants}

For the identification of human factors related with accidents, the participation of 21 members of safety group was required; among them $81.3 \%$ were male and $13.7 \%$ female. In relation to their age it was observed that $57.1 \%$ were over 41 years old, while the $42.9 \%$ were in a range of 30 to 40 years old. The seniority of most of participants was more than 10 years for almost $80 \%$ of participants and between 5 and 10 years for the rest or the sample. Seniority in actual position during the study was between 7 and 15 years for more than $50 \%$ of the participants, 6 years or less for $33.3 \%$ of them, and over 16 years for only $9.5 \%$ of participants. The dominant profession among participants was engineering, with $42.9 \%$ followed by business adminis- 
tration with $28.6 \%$, only one of participants had a master degree and only one of them was a medicine doctor. It was observed that $19 \%$ of among participants have no particular profession and their education level was highschool.

During the second stage of the study about the classification of human factors, all the 21 initial members of the safety group the study were invited, but only 12 of them continue collaborating with the research. Among them 11 were male and 1 female. $41.7 \%$ of participants had less than 40 years old and $58.3 \%$ between 41 and 46 . Seniority in the company presented $33.3 \%$ in the range of 11 to 14 years, $33.4 \%$ between 15 and 22 , while only $16.7 \%$ greater than or equal to 23 years. Regarding to seniority position, there was $41.7 \%$ between 5 and 10 years, only $16.6 \%$ in the range of 11 and 25 years, and $41.7 \%$ less than 5 years. In the case of the profession dominated engineer with a $50 \%, 25 \%$ for business administration degree and $25 \%$ do not have any profession; their education level was high school. In relation to the position occupied by the participants in the company was found a prevalence of $58.3 \%$ for supervisors, followed by $16.75 \%$ for managers, the superintendent, technical and health coordinator formed $24.9 \%$ of the sample.

\subsection{Cultural Domain analysis}

The results of the first free listing confirmed the central assumption of the CCT, which establishes the existence of a body of knowledge, supported by a specific cultural domain. This assumption refers to the existence of experts with greater knowledge than others. The cultural domain consisted of 70 items, some of these presented low frequencies. According to Weller, the researcher must verify that all items are known by the informants [34], the results of the second free listing confirmed it; most of the items presented high frequencies, but only two of them low.

\subsection{Cluster Analysis and Multidimensional Scaling}

The cluster analysis was applied for 2, 4 and 8 piles with partitions of 2, 3, 4 and 5, others were unnecessary. Partition 2 was choosing because it presents greater internal cohesion, as the items showed more similarity between them. A graphical representation through a dendrogram was performed. This chart was useful to evaluate the clusters homogeneity and facilitate the decision regarding the op- timal number of these. The dendrogram shows four clusters that formed categories. Informants assigned names to these categories. Thus, human factors were classified into the categories: human error, unsafe conditions, personal factors and organizational factors.

MSD was used as a complementary technique to cluster analysis and helped to confirm the findings in it. Lower values for the Stress coefficient were 0.096 and 0.107 for 4 and 8 piles respectively. According to the interpretation criteria proposed by Kruskal [16], the lowest coefficient of Stress (0.096) would be the case with a number of piles equal to 4 and the adjustment to the MDS model would be between good and acceptable, while for the case with 8 piles Stress value 0.107 adjustment of the MDS model was considered fair to poor. In agreement to the criterion of goodness of fit (Stress), MDS model with 4 piles provides the best fit and therefore the results were verified with the cluster analysis.

\subsection{Cultural Consensus Analysis}

Table 1 shows the results of cultural consensus analysis among safety group, obtained from the data of pile sort application with 4 piles. The data fitted the cultural consensus model and did not present clear evidence of subcultural variation among participants, suggesting a coherent cultural domain.

Table 1

Cultural consensus analysis of safety group

\begin{tabular}{lccc}
\hline Informant & $\begin{array}{l}\text { Cultural } \\
\text { competence }\end{array}$ & Informant & $\begin{array}{c}\text { Cultural } \\
\text { competence }\end{array}$ \\
\hline 1 & 0.569 & 7 & 0.734 \\
2 & 0.668 & 8 & 0.836 \\
3 & 0.836 & 9 & 0.683 \\
4 & 0.593 & 10 & 0.488 \\
5 & 0.579 & 11 & 0.684 \\
6 & 0.646 & 12 & 0.789 \\
\hline Eigenvalue ratio & 5.607 & Mean & 0.6754 \\
Eigenratio & 4.449 & Tipycal.D & 0.1093 \\
Group agreement** & 0.4561 & & \\
\hline
\end{tabular}

According to Weller and Romney [31] the ratio of the eigenvalues (eigenratio), indicate whether there is a level of agreement Cultural Consensus Analysis sufficient among the participants to conclude the existence of a shared model. The general rule is that the first eigenvalue must be at least 3 times greater than the second, i.e. the ratio of the eigenvalues is greater than 3 , indicating the existence of a shared model. 
An eigenratio of 4.449 validates the existence of a shared model, a group agreement to 0.4561 and a cultural competence average of 0.6754 , so according to Weller [34], the cultural pattern is strong.

In relation to the validity and reliability of the classification obtained a sample sizes of 12 and an average of cultural competence $n$ relation to the validity and reliability of the classification obtained can set you up with sample sizes of 12 and an average of 0.6 cultural competence, properly classified at

least $90 \%$ of the items to a confidence level of $99 \%$. on relation to the validity and reliability of the classification obtained can set you up with sample sizes of 12 and an average of 0.6 cultural competence, properly classified at least $90 \%$ of the items to a confidence level of $99 \%$.f 0.6 , is properly classified at least $90 \%$ of the items to a confidence level o $99 \%$.

\subsection{Human Factors Categories}

In the Tables 2, 3 and 4 are presented categories of personal factors, human error, unsafe conditions, and organizational factors respectively. These tables include the code used in the identification of the element during the application of the technique pile sort, items mentioned by the participants in the free listing and the percentage of participants who mentioned the item in the category.

Table 2

Personal factors category

\begin{tabular}{clc}
\hline Code & Personal factors & $(\%)$ \\
\hline 56 & Using hands jewelry & 88 \\
68 & Talking while working & 88 \\
45 & Fatigue or tiredness & 88 \\
22 & Health of the person & 62 \\
47 & Lack of care or interest in doing their work & 82 \\
34 & The worker's inappropriate behavior & 57 \\
24 & Personal problems & 62 \\
38 & Family problems & 57 \\
54 & Indiscipline & 88 \\
69 & Talking on the phone while working & 88 \\
50 & Drowsiness by not sleeping properly & 82 \\
62 & Recklessness & 88 \\
58 & Excessive intake of alcohol or drugs before & 64 \\
& reporting to work & \\
\hline
\end{tabular}

Table 2 shows the main expressions related to personal factors found by participants. It can be observed that attitudes related with personal appear- ance, phone talking, fatigue, recklessness and workers' indiscipline are the ones with higher percentage of use.

Table 3 shows the main expressions related to human error found by participants. It can be seen that the top tree expression are related to distractions or careless from operators, playing in workspaces and lack or personal protection equipment.

Table 3

Human error category

\begin{tabular}{|c|c|c|}
\hline Code & Human error & $\%$ \\
\hline 66 & Improper handling of heavy objects & 82 \\
\hline 15 & $\begin{array}{l}\text { Trying to save time in developing their } \\
\text { operation }\end{array}$ & 76 \\
\hline 13 & $\begin{array}{l}\text { The operator does not respect rules and } \\
\text { procedures safety }\end{array}$ & 76 \\
\hline 52 & Component on the floor & 82 \\
\hline 4 & $\begin{array}{l}\text { Failure to follow work instructions (me- } \\
\text { thod) }\end{array}$ & 85 \\
\hline 18 & Disregarding security systems & 76 \\
\hline 12 & Overconfidence & 76 \\
\hline 3 & $\begin{array}{l}\text { Distraction or carelessness of the operator } \\
\text { to perform its task }\end{array}$ & 90 \\
\hline 2 & Workers play in workspaces & 90 \\
\hline 57 & Misinterpretation of work instruction & 70 \\
\hline 1 & $\begin{array}{l}\text { Perform tasks without personal protective } \\
\text { equipment (gloves) }\end{array}$ & 90 \\
\hline 28 & $\begin{array}{l}\text { Manage the sharp terminal cable without } \\
\text { gloves }\end{array}$ & 57 \\
\hline 40 & Do not use the right tool & 52 \\
\hline 26 & Two workers operating equipment & 62 \\
\hline 60 & Remove with fingers stuck terminals & 82 \\
\hline 29 & Operating equipment without knowledge & 57 \\
\hline 37 & Work without safety guards in machines & 52 \\
\hline 63 & Unauthorized use of knives & 70 \\
\hline
\end{tabular}

Table 4 shows the main expression related to organizational factors found by participants. In this, it can be seen that lack of attention to preventive safety, monotonous and boring work, insufficient safety training are the ones with the higher percentage of use. In relation with unsafe conditions, it can be observed that the lack of safety guards on equipment is the one with the higher percentage of use.

\section{Conclusions}

In relation with the human error category; results coincide with Rasmussen [20], Reason [19], and Shapell [11] taxonomies. 
Table 4

Organizational factors and unsafe acts categories

\begin{tabular}{|c|c|c|}
\hline Code & Organizational factors & $(\%)$ \\
\hline 30 & Insufficient planning to improve safety aspects & 57 \\
\hline 19 & The safety culture of workers and employees hasn't a strong focus on accident prevention & 76 \\
\hline 17 & Repetitive motions in operations & 76 \\
\hline 53 & There is an overload of work & 76 \\
\hline 9 & The budget for security is insufficient & 80 \\
\hline 35 & The stressful working environment & 57 \\
\hline 61 & Lack motivation to comply with the procedures and rules to prevent accident & 76 \\
\hline 33 & Involvement of staff in safety is insufficient & 57 \\
\hline 11 & Lack of personal protective equipment (gloves and thimbles) & 80 \\
\hline 44 & Lack of focus in ergonomics & 88 \\
\hline 49 & Slow response to solving problems concerning safety & 88 \\
\hline 59 & Little attention to preventive security & 94 \\
\hline 67 & Lack of training in jobs with risk of accidents & 80 \\
\hline 70 & Monotonous and bored work & 90 \\
\hline 20 & The needs of production cause the release of unsafe equipment & 62 \\
\hline 51 & The procedures and methods do not consider security aspects & 70 \\
\hline 65 & The objectives of the company are many and change fast & 47 \\
\hline 39 & Lack of communication in aspects of accident prevention & 57 \\
\hline 42 & Security best practices carried out in other companies are not shared & 52 \\
\hline 32 & The working methods are confusing and without safety instructions & 57 \\
\hline 7 & Safety training insufficient & 88 \\
\hline 25 & Pressure by the operator for production delays & 62 \\
\hline 21 & Assignment of personnel without required training & 62 \\
\hline 43 & The low involvement of supervisor in monitoring and risk detection & 94 \\
\hline 41 & Poco seguimiento a la correcion de las condiciones inseguras & 52 \\
\hline Code & Unsafe conditions & $(\%)$ \\
\hline 16 & Damaged tools & 71 \\
\hline 55 & Hot zones in equipment & 58 \\
\hline 6 & Poor condition equipment for lack of maintenance & 85 \\
\hline 36 & Workspaces reduced & 57 \\
\hline 8 & Long, hard and misplaced bolts & 80 \\
\hline 27 & Operating speed above the standard or cycle time & 57 \\
\hline 10 & Poor ergonomic design of the workstation & 80 \\
\hline 46 & Inadequate design of tools & 82 \\
\hline 48 & Lack of auditive and visual preventive displays & 47 \\
\hline 14 & Lack of lighting in work areas & 76 \\
\hline 5 & Lack of safety guards on equipment & 85 \\
\hline 23 & Safety guards with improper design in press & 62 \\
\hline 31 & Workstation with sharp edges & 57 \\
\hline 64 & Incorrect use of chemicals & 52 \\
\hline
\end{tabular}

Most of the items mentioned in this category corresponded to violations, also mistakes and lapsus were found.

Categories corresponding to unsafe conditions, individual factors and organizational factors present a high agreement with the framework of human error of Sharit and Gables [18] and Reason [19].

Overall, the Cultural Consensus methodology was effective in identifying and categorizing items which 
correspond to active and latent accidents causes according Swiss Chesse Model [19].

The used methodology presents advantages when very complex problems must be explored and the

Future efforts can be focused on designing a specific taxonomy to classify and analyze human factors in occupational accidents for manufacturing industry. It should be useful as a starting point for implementing preventive actions based on contributing factors accidents in assembly industry of automotive harnesses.

\section{References}

[1] A.Boquet, C. Detwiler, S.Shappell.A human factor s analysis of U.S.emergency medical transport accidents. Air Medical Journal 23 (5), (2004) pp. 34-34

[2] A. Harris, Erring of the side of danger. Occupational Health, 56(2004), 24-27.

[3] A.J., Ross, Wallace, B, and J.B. Davies. Technical note: measurement issues in taxonomic reliability. Safety Science, 42(2004), 771-778.

[4] A. K, Romney, W. H. Batchelder, and S. C. Weller. Recent applications of consensus theory. American Behavioral Scientist 31 (1987), 163-77.

[5] A.K Romney. Culture consensus as a statistical model. Current Anthropology 40 (1999), S103-15.

[6] A. K Romney, S. C. Weller, and W. H. Batchelder. Culture as consensus: A theory of culture and informant accuracy. American Anthropologist 88(1986), 313-38.

[7] A.Tashakkori and J.W. Creswell A new era for mixed methods. Journal of Mixed Methods Research, 1(2007), 3-7.

[8] A.W, El Bardissi, D.A.Wiegmann, J.A, Dearani, R.C., Daly, T.M Sundt. Application of the human factors analysis and classification system methodology to the cardiovascular surgery operating room. The Annals of Thoracic Surgery 83 (4), (2007), 1412-1419.

[9] C. A. Steinhaus-Donham, Illness beliefs of deaf senior citizens. Ann Arbor, MI: University Microfilms (1987).

[10]C. Webster, A. L. Iannucci and A.K. Romney, Consensus Analysis of Personality Traits. Field Methods 14 (2002), 46 DOI: $10.1177 / 1525822$ X0201400104

[11]D. A. Wiegmann and S. A. Shappell. Assessing the reliability of the human factors analysis and classification system (HFACS).Aviation, Space, and Environmental Medicine, 72(2003), 1006-1016.

[12]D.Brewer, A. K. Romney, and W. H. Batchelder. Consistency and consensus: A replication. Journal of Quantitative Anthropology 3(1991), 195-205.

[13]D.D Woods and R.I Cook. Incidents - Markers of Resilience or Brittleness? In Hoolnagel, E.Woods and N.Levenson. (Eds), Resilience Engineering: Concepts and Precepts, 2006 p.p. 6976.Aldershot, UK; Ashgate.

[14]F.J.Milligan. Establishing a culture for patient safety-the role of education. Nurse Education Today. 27 (2), (2007), 95-102. description of results can be better explained for future applications.

[15]Health and Safety Executive. (1989).Human Factors in Industrial Safety. London: HMSO

[16]J. B.Kruskal and M.Wish. Multidimensional scaling. Sage University Papers Series on Quantitative Applications in the Social Sciences .Thousand Oaks, CA: Sage, (1978).

[17] J.Leplat and J.Rasmussen. Analysis of human errors in industrial incidents and accidents for the improvement of work safety. Accident Analysis and Prevention, 16(1984), 77-88.

[18]J. Sharit and C.Gables, Human error. En G.Salvendy (Ed), Handbook of Human Factors and Ergonomics (2006).708760.U.S.A: John Wiley \& Sons.inc.

[19]J.T. Reason. Human Error. Cambridge: Cambridge University Press, (1990).

[20] J. Rasmussen. Human errors: taxonomy for describing human malfunction in industrial installations. Journal of Occupational Accidents, 4(1982), 311-333.

[21]K. A Jameson and A. K. Romney.Consensus on semiotic models of alphabetic systems. Journal of Quantitative Anthropology 2(1990), 289-303.

[22]K. A Jameson. An empirical investigation of semiotic characterizations of alphabetic systems. Ann Arbor, MI: University Microfilms (1989).

[23]L. C. Garro. Explaining high blood pressure: Variation in knowledge about illness. American Ethnologist 15(1988), 98 119.

[24]M.Celik and S. Cebi. Analytical HFACS for investigating human errors in shipping accidents. Accident Analysis and Prevention, 41(2009), 66-75.

[25]P.D. Baker and J.K Krokos. Development and Validation of Aviation Causal Contributors for Error Reporting System (ACCERS). Human Factors, 49(2007) (2), 185-199.

[26] R.DeJordy, S. Borgatti, Ch.Roussin, and D. Halgin. Visualizing Proximity Data. Field Methods; 19, No. 3(2007), 239-263.

[27]R. G D'Andrade. Culture is not everything. In E. L. CerroniLong (Ed.), Anthropological theory in North America 1999, pp. 85-103. Westport, CT: Bergin \& Garvey. Shore, B. 1996 Culture in mind: Cognition, culture, and the problem of meaning. New York: Oxford, University Press. University Press

[28]R. G D'Andrade. Cultural meaning systems. In R. A. Schweder \& R. A. Levine (Eds.), Culture theory: Essays on mind, self, and emotion .1984.pp. 88-119. Cambridge, UK: Cambridge University Press.

[29] S. A Shappell and D. Wiegmann.US naval aviation mishaps: differences between single and dual piloted aircraft. Aviation, space, and Environmental Medicine.67 (1999), 65-69.

[30] S.Borgatti, Visual ANTHROPAC version 1.02.35. Natick, MA: Analytic Technologies, (2003).

[31]S. C Weller and A. K Romney. (1988). Systematic data collection. Thousand Oaks, CA: Sage.

[32] S.A Shappell, C. Detwiler, K. Holcomb, C.Hackworth, Boquet, A. and D.A. Wiegmann. Human error and commercial aviation accidents: an analysis using the human factors analysis and classification system. Human Factors43 (2) (2007), 227242. 
[33]S.Reinach and A.Viale.Application of the human error framework to conduct train accident/incident investigations. Accident Analysis and Prevention, 38(2006), 396-406.

[34]S.R Weller. Cultural Consensus Theory: Applications and Frequently Asked Questions. Field Methods, 19, 4 (2007), 339-368
[35] T.M.Baysari S.A. McIntosh and J. R Wilson. Understanding the human factors contribution to railway accidents in Australia. Accidents Analysis and Prevention, 40(2008), 1750-1757. 